\title{
Ultrarote Eigenfrequenzen der Chlorate, Bromate, Jodate.
}

Von Clemens Schaefer und Martha Schubert in Marburg.

Mit vier Abbildungen. (Eingegangen am 23. August 1921.)

Unsere früheren Messungen $\left.{ }^{1}\right)$ bei den Karbonaten $\left(\mathrm{CO}_{3}\right.$-Gruppe) und den Nitraten $\left(\mathrm{NO}_{3}\right.$-Gruppe) hatten ergeben, daß der $\mathrm{CO}_{3}$-Gruppe folgende Maxima der Reflexionen eigentümlich sind 2):

$$
\lambda_{1}=6,5 \mu, \quad \lambda_{2}=11,5 \mu, \quad \lambda_{8}=14,5 \mu,
$$

und ebenso der $\mathrm{NO}_{3}$-Gruppe die folgenden:

$$
\lambda_{1}=7,5 \mu, \quad \lambda_{2}=12,5 \mu, \quad \lambda_{3}=15,0 \mu .
$$

Diese auffallende Übereinstimmung - die Maxima der $\mathrm{NO}_{3}$-Gruppe sind lediglich gegenüber denen der $\mathrm{CO}_{3}$-Gruppe etwas nach längeren

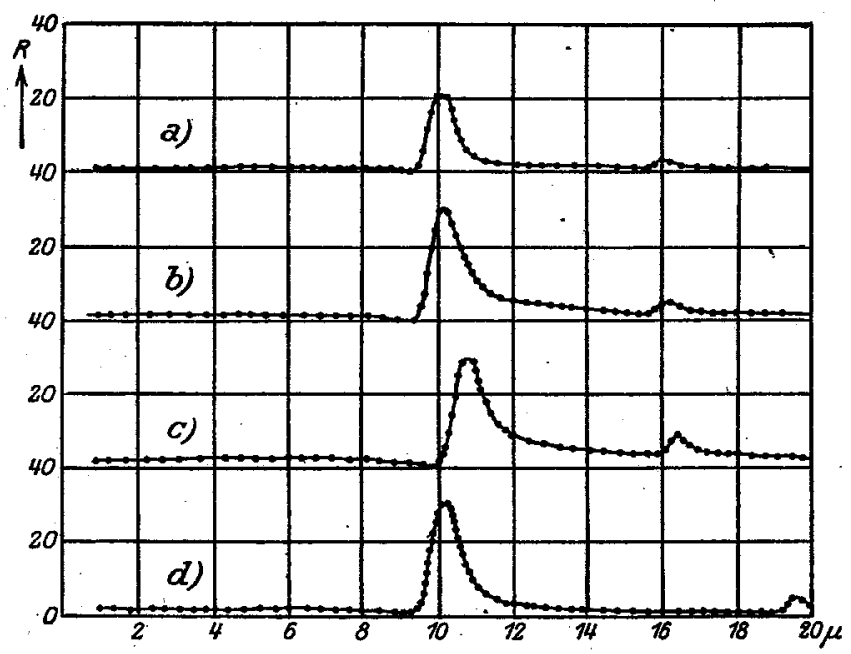

Fig. 1.

a) Natriumchlorat, b) Kaliumchlorat, c) Bilberchlorat, d) Baryumchlorat.

Wellenlängen verschoben, entsprechend dem höheren Atomgewicht des $\mathbf{N}$ gegenüber dem $\mathrm{C}$ - legte es nahe, die entsprechende Untersuchung auch bei den Chloratep $\left(\mathrm{ClO}_{3}\right.$-Gruppe), den Bromaten $\left(\mathrm{BrO}_{3}\right.$ Gruppe) und den Jodaten ( $\mathrm{JO}_{3}$-Gruppe) auszaführen. Die Messungen sind bereits seit mehr als Jahresfrist beendet; ihre Veröffentlichung

1) Cl. Schaefer und M. Schubert, Ann. d. Phys. 50, 283, 1916; ebenda 55, 577, 1918.

2) Die Wellenlängenangaben sind nur ungefähre: 
hat sich wegen des Weggangs des einen vion uns von Breslau bis jetzt unliebsam verzögert.

Zar Untersuchung gelangten die Substanzen, die in der ersten Kolumne der folgenden Tabelle aufgeführt sind; die zweite Kolumne enthält die Angabe des Kristallsystems, die dritte die Angabe, welcher Schnitt untersucht wurde, die vierte Bemerkungen, die sonst zur Charakteristik von Wert sind, die fünfte endlich die beobachteten Reflexionsmaxima.

\begin{tabular}{|c|c|c|c|c|}
\hline Name & $\begin{array}{l}\text { Kristall- } \\
\text { system }\end{array}$ & $\begin{array}{l}\text { Untersuchter } \\
\text { Schnitt }\end{array}$ & Bemerkungen & $\begin{array}{l}\text { - Beob, } \\
\text { Reflexionsmaxima }\end{array}$ \\
\hline $\mathrm{NaClO}_{3}$ & regulär & - & $\begin{array}{c}\text { Platte aus kleinen } \\
\text { Kristallen } \\
\text { zusammengesetzt }\end{array}$ & $\begin{array}{l}\lambda_{1}=10,04 \mu \\
\lambda_{2}=16,04 \mu\end{array}$ \\
\hline $\mathrm{KClO}_{3}$ & monoklin & - & $\begin{array}{c}\text { gepreßtes } \\
\text { Kristallpulver }{ }^{1} \text { ) }\end{array}$ & $\begin{array}{l}\lambda_{1}=10,12 \mu \\
\lambda_{2}=16,22 \mu\end{array}$ \\
\hline $\mathrm{AgClO}$ & tetragonal & 一 & $\begin{array}{c}\text { gepreBtes } \\
\text { Kristallpulver }{ }^{1)}\end{array}$ & $\begin{array}{l}\lambda_{1}=10,76 \mu \\
\lambda_{2}=16,38 \mu\end{array}$ \\
\hline $\begin{array}{l}\mathrm{Ba}\left(\mathrm{ClO}_{3}\right)_{2} \\
+1 \mathrm{H}_{2} \mathrm{O}\end{array}$ & $\underset{\text { prismatisoh }}{\text { monoklin }}$ & $\begin{array}{c}\text { Sehnitt H } \\
\text { Symmetrie- } \\
\text { ebene }\end{array}$ & $\begin{array}{l}\text { Platte aus kleinen } \\
\text { Kristallen } \\
\text { zusammengesetzt }\end{array}$ & $\begin{array}{l}\lambda_{1}=10,24 \mu \\
\lambda_{2}=19,6 \mu\end{array}$ \\
\hline $\mathrm{NaBrO}$ & regulär & - & $\begin{array}{c}\text { Schnitt aus einem } \\
\text { großen Kristall }\end{array}$ & $\lambda_{1}=12,24 \mu$ \\
\hline & 一 & 一 & $\underset{\text { Kristallpulver }}{\text { gepreßtes }}$ & $\begin{array}{l}\lambda_{i}^{\prime}=12,62 \mu \\
\lambda_{1}^{\prime \prime}=13,10 \mu\end{array}$ \\
\hline $\begin{array}{l}\mathrm{Cd}\left(\mathrm{BrO}_{3}\right)_{2} \\
\quad+2 \mathrm{H}_{2} \mathrm{O}\end{array}$ & rhombisch & $\begin{array}{l}\text { Schnitt } \mathrm{H} \\
\mathfrak{a} \mathfrak{c}-\text { Ebene }\end{array}$ & $\begin{array}{l}\text { Platte aus kleinen } \\
\text { Kristallen } \\
\text { zusammengesetzt }\end{array}$ & $\begin{array}{l}\lambda_{1}^{\prime}=12,32 \mu \\
\lambda_{2}^{\prime}=13,20 \mu\end{array}$ \\
\hline $\begin{array}{r}\mathrm{Ba}\left(\mathrm{BrO}_{3}\right)_{2} \\
+1 \mathrm{H}_{2} \mathrm{O}\end{array}$ & monoklin & $\begin{array}{l}\text { Behnitt H } \\
\text { ac-Ebene }\end{array}$ & $\begin{array}{l}\text { Platte aus kleinen } \\
\text { Kristallen } \\
\text { zusammengesetzt }\end{array}$ & $\begin{array}{l}\lambda_{i}^{\prime}=12,3 \mu \\
\lambda_{1}^{\prime \prime}=12,64 \mu\end{array}$ \\
\hline $\mathrm{KJO}_{3} \cdot$ & - & 一 & $\begin{array}{c}\text { gepreBtes } \\
\text { Kristall pulver }{ }^{1} \text { ) }\end{array}$ & $\begin{array}{l}\lambda_{1}^{\prime}=12,48 \mu \\
\lambda_{1}^{\prime \prime}=13,1 \ddot{6} \mu\end{array}$ \\
\hline
\end{tabular}

Die Verhältnisse erkennt man am einfachsten durch Betrachten der Fig. 1 bis 3, die ohne weitere Erklärung verständlich sind.

Im einzelnen ist noch folgendes zu bemerken:

Drei Maxima (wie bei den Karbonaten und Nitraten) sind hier nicht beobachtet. Das braucht aber nicht gegen die Voraussetzung za sprechen, von der wir oben ausgegangen sind: denn wegen des höheren Atomgewichtes von $\mathrm{Cl}, \mathrm{Br}$ und $\mathrm{J}$ müssen die Wellenlängen sämtlich nach längeren Wellen verschoben sein. In. der Tat finden wir ja auch das erste Maximum der Chlorate hier erst bei etwa 10,5 $\mu$, das der Bromate bei etwa $12 \mu$ und das des einen untersuchten Jodats bei etwa $12,5 \mu$. Bei den Chloraten ist noch ein zweites Maximum

1) Vgl. hierzu: Cl. Schaefer n. M. Schubert, Ann. d. Phys. 55, 581, 1918. 
bei etwa $16 \mu$ vorhanden; nimmt man an, daß dieses dem zweiten Maximum der Karbonate bei $11,5 \mu$ und dem der Nitrate bei $12,5 \mu$ entspricht, so ist auch hier die starke Verschiebung nach rechts im Einklang mit unserer Auffassung. Bei den Bromaten und Jodaten

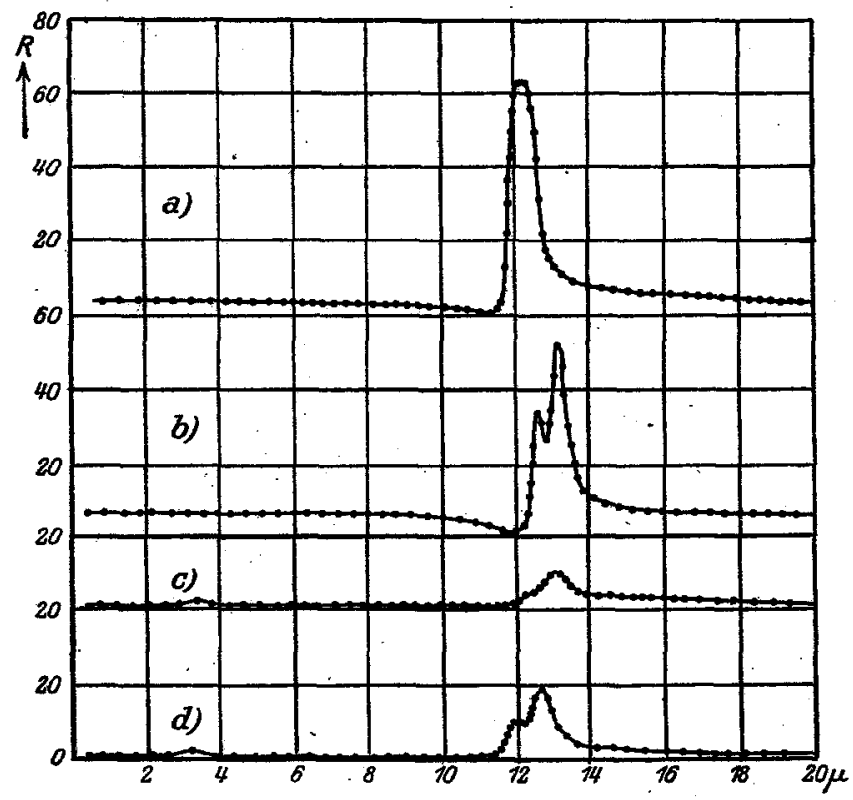

Fig. 2.

a) Natriumbromat, b) Silberbromat, c) Cadmiumbromat, d) Baryumbromat.

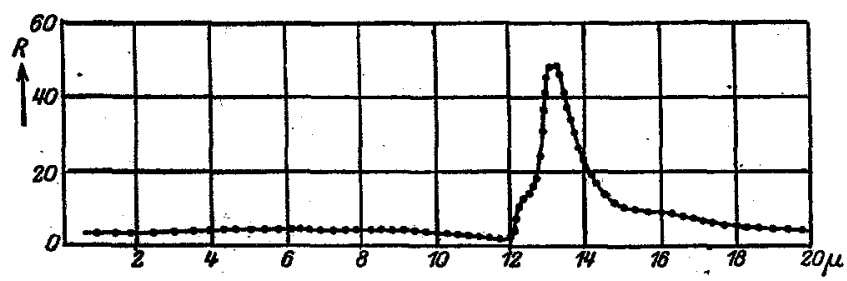

Fig. 3. Kaliumjodat.

wäre dann im selben Sinne anzunehmen, daß das zweite Maximum schon jenseits $20 \mu$ liegt, und ebenso würde es dann mit dem dritten Maximum sein müssen, daß dann sowohl bei den Chloraten als auch bei den Bromaten and Jodaten jenseits $20 \mu$ herausgerückt wäre.

Natürlich bieten die hier angefübrten Tatsachen keinen Beweis, da $B$ es sich wirklich so verhält und eine weitere Untersuchung in dieser Richtung ist notwendig. DaB aber wirklich das erste Maximum 
312 Schaefer u. Schubert, Ultrarote Eigenfrequenzen der Chlorate usw.

der Chlorate, Bromate und Jodate innerlich mit dem entsprechenden ersten Maximum der Karbonate und Nitrate zusammengehört, kann man durch folgende Uberlegung noch weiter stützen: Man sollte in diesem Falle erwarten, daß èin gesetzmäßiger Zusammenhang zwischen

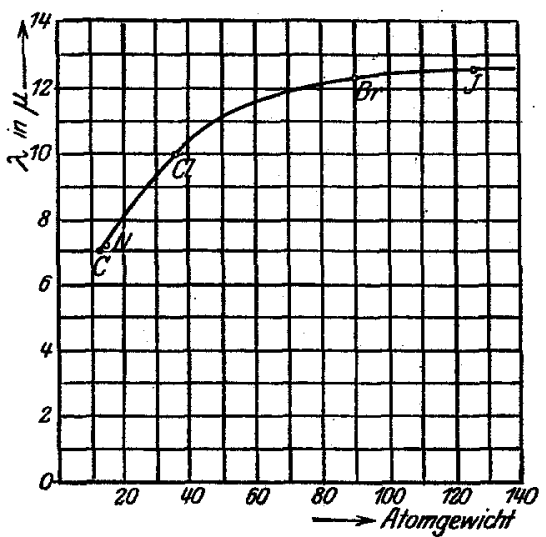

Fig. 4. der Lage dieser ersten Maxima und den Atomgewichten von $\mathrm{C}$, $\mathrm{N}, \mathrm{Cl}, \mathrm{Br}, \mathrm{J}$ vorhanden wäre. Dies scheint tatsächlich der Fall zu sein, wie ein Blick anf die Fig. 4 lehrt, in der die Atomgewichte als Abszissen, die Eigenwellenlängen des ersten Maximums als Ordinaten aufgetragen sind. Auch die zweiten Reflexionsmaxima, gegen die Atomgewichte aufgetragen, liegen auf einer glatten Kurve; nur ist diese letztere nicht sehr beweiskräftig, weil eben nur drei Punkte derselben bestimmt sind. Jedenfalls aber dürfen wir sagen, daß die Tatsachen nicht im Widerspruch mit unserer Auffassung stehen.

In einzelnen zeigen die Fig. 1 bis 3 manches Interessante, worauf hier wenigstens hingewiesen sei. Bei jeder Gruppe (z. B. bei den Chloraten) findet im allgemeinen wieder eine Verschiebung der Lage der Maxima mit dem Atomgewicht des Metallions (Na, K, $\mathrm{Ag}, \mathrm{Ba}$ ) statt. Doch findet sich z. B. das erste Maximum des Silberchlorats stärker nach rechts gerückt, als das des Bariumchlorats; etwas Analoges findet bei den Bromaten statt. Doch sei hier nur die Tatsache konstatiert, ohne daß wir anf eine Diskussion der Gründe dafür näher eingeben wollen, die einer späteren Untersuchung vorbehalten bleiben soll.

Soweit möglich, sind die doppelbrechenden unter den obigeu Kristallen auch im polarisierten Lichte untersucht worden. Hier ergab sich stets, daß eine beim regulären Kristall einfache Eigenschwingung sich beim einachsigen Kristall in zwei, beim zweiachsigen Kristall in drei Eigenschwingungen aufspaltet, die den verschiedenen Schwingungsrichtungen im Kristall zugeordnet sind, genau, wie wir es früher gefunden haben. Ein genaueres Eingehen hierauf ist daher unnötig.

Als Resultat ist also die Existenz von bestimmen Eigenfrequenzen festzustellen, die bzw. der $\mathrm{ClO}_{3}^{-}$, der $\mathrm{BrO}_{3}$ - und der $\mathrm{JO}_{3}$-Gruppe zuzuschreiben sind.

Marburg und Breslau, im August 1921. 